\title{
LOW-DEGREE $P$-MODE SOLAR CYCLE TRENDS FROM BISON DATA
}

\author{
W. J. CHAPLIN, Y. ELSWORTH. G. R. ISAAK. C. P. MCLEOD AND \\ B. A. MILLER \\ School of Physics 83 Astronomy, University of Birmingham \\ Edgbaston, Birmingham B15 2TT UK \\ E-mail: wjc@star.sr.bham.ac.uk \\ AND \\ R. NEW \\ School of Science \& Mathematics, Sheffield Hallam University \\ Sheffield, S1 $1 \mathrm{WB}$
}

An in-depth discussion of the analysis presented here can be found in an up-coming paper (Chaplin et al. 1997).

\section{Trends at frequencies up to $\sim 3900 \mu \mathrm{Hz}$}

In order to investigate the solar-cycle dependence of the low-degree $p$-mode frequencies, we have analysed eighteen 4-month frequency spectra generated from BiSON Doppler velocity residuals collected between 1991 January 01 and 1997 January 05 . These data cover the falling phase of solar activity cycle 22 , up to the cycle $22 / 23$ boundary. Fig. 1(a) shows frequency shifts, averaged over two orders in $n$, up to $\sim 3900 \mu \mathrm{Hz}$ - as derived from the analysis of the 4-month spectra - normalised to unit change in the $10.7-\mathrm{cm}$ radio flux. The dashed line lying above the BiSON data is a fit to the BBSO 1989-minus-1986 frequency shifts, for $4 \leq \ell \leq 140$ (Libbrecht \& Woodard. 1991). The dotted line passing through the data corresponds to the bestscaled fit of the BBSO data to the BiSON data - the best-fit requires the BBSO data to be scaled by $0.71 \pm 0.03$. This is reasonably consistent - as expected - with the mean, overall ratio of the inverse mode masses of those data used in the BiSON and BBSO analyses $(\approx 0.67)$. Fig. $1(\mathrm{~b})$ shows the BiSON activity-normalised frequency shifts. plotted as a function of inverse mode mass - here, as anticipated, there is a clear correlation between the variables.

\section{Trends at frequencies above $\sim 4000 \mu \mathrm{Hz}$}

At high frequencies we have fitted the low- $\ell$ pairs to a single-Lorentzian model in 4 and 8 -month averages of short. 7.6-d spectra. The bottom figure below shows the solarcycle dependence - again normalised to unit change in the 10.7-cm flux - derived 

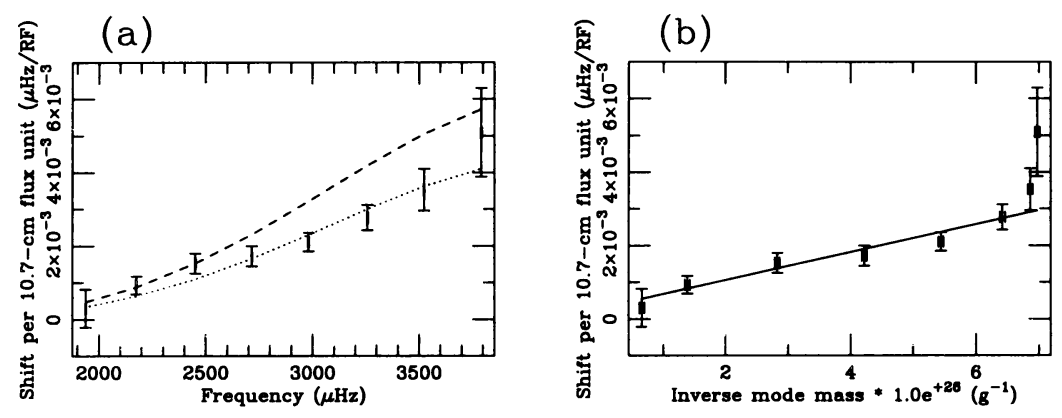

Figure 1. Solar-cycle dependence of fitted BiSON modes at frequencies up to $\approx 3900 \mu \mathrm{Hz}$. In (a) - plotted as a function of frequency. The dashed line lying above the BiSON data is a fit to the BBSO 1989-minus-1986 frequency shifts, for $4 \leq \ell \leq 140$. The dotted line passing through the data corresponds to the best-scaled fit of the B $\overline{B S O}$ data to the BiSON data. And in (b) - plotted as a function of inverse mode mass.

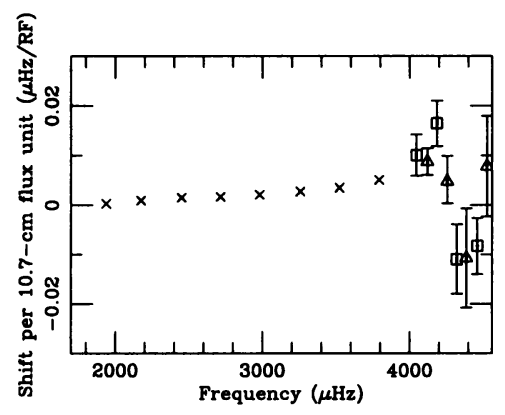

Figure 2. Solar-cycle dependence at high frequency - again normalised to unit change in the $10.7-\mathrm{cm}$ flux - derived from single Lorentzian fits to 4-month averaged $\ell=2 / 0$ pairs (triangular symbols with errors) and $\ell=3 / 1$ pairs (square symbols with errors). The crosses are the data from Fig. 1(a).

from single Lorentzian fits to 4 -month averaged $\ell=2 / 0$ pairs (triangular symbols with errors) and $\ell=3 / 1$ pairs (square symbols with errors). We also include data from Fig. 1(a) as a lower-frequency reference. If we consider the extracted solar-cycle coefficients, the data are suggestive of a turnover and possible sign reversal (a straightline fit gives a gradient significant at the $3 \sigma$ level). Clearly, one must be cautious (e.g.: possible systematic complications for the $\ell=3 / 1$ fits; see Chaplin et al., 1997).

\section{References}

Chaplin W. J.. Elsworth Y., Isaak G. R., McLeod C. P., Miller B. A. and New R., 1997. MNRAS. submitted

Libbrecht K. G. \& Woodard M. F., 1991, Science. 253, 152 